\title{
Validación español-argentina del cuestionario de transición a la atención médica del adulto en adolescentes con enfermedades crónicas
} Validation of the Argentine Spanish version of Transition Readiness
Assessment Questionnaire for adolescents with chronic conditions

\author{
Dra. Florencia González ${ }^{a}$ Dra. Mariana Roizen ${ }^{a}$, \\ Dra. María de las Mercedes Rodríguez Celin ${ }^{a}$, Dra. Carmen De Cunto ${ }^{b}$, \\ Dr. Alfredo Eymann ${ }^{b}$, Dr. Roberto Mato ${ }^{a}$, Dra. Patricia García Arrigoni ${ }^{a}$, \\ Dra. Raquel Staciuk ${ }^{a}$ Lic. Florencia Ugo y Dra. Virginia Fano ${ }^{a}$
}

a. Hospital Nacional de Pediatría Garrahan.

b. Departamento de Pediatría del Hospital Italiano de Buenos Aires.

Correspondencia: Dra. Florencia González, gonzalezfl@yahoo.com.ar

Financiamiento: La Dra. Florencia González recibió una beca de investigación otorgada por la Coordinación de Investigación y financiada por la Fundación del Hospital de Pediatría "Prof. Dr. J. P. Garrahan" durante los años 2014 ("Construcción de un programa de transición para pacientes adolescentesadultos jóvenes con enfermedades crónicas en el Hospital Garrahan: sentando las bases") y 2015 ("Ampliación de la validación y evaluación de la utilidad del TRAQ 5.0 y difusión de la problemática de la transición en el Hospital Garrahan").

Conflicto de intereses: Ninguno que declarar.

Recibido: 10-6-2016 Aceptado: 26-9-2016

\section{RESUMEN}

Introducción. El pasaje de adolescentes con enfermedades crónicas al seguimiento como adultos es un proceso complejo y creciente. Los pacientes necesitan adquirir conocimientos y habilidades que aseguren la continuidad de su cuidado. Elobjetivo fuellevar a cabola validación del instrumento Transition Readiness Assessment Questionnaire (TRAQ) 5.0, versión en español argentino, en adolescentes y adultos jóvenes con enfermedades crónicas.

Población y métodos. Estudio descriptivo, transversal y cuantitativo. Se incluyeron pacientes mayores de 14 años con enfermedad crónica atendidos en el Hospital Garrahan. El TRAQ incluye 20 ítems en 5 subescalas (medicación, asistencia a citas, seguimiento de problemas de salud, comunicación con profesionales, manejo de actividades cotidianas) y se responde de modo autoadministrado. Los pacientes completaron el TRAQ, una encuesta de opinión sobre su uso y otra escala de autopercepción de autonomía; sus médicos, una escala sobre el compromiso de la enfermedad. Se registraron variables sociodemográficas, clínicas y relacionadas con el TRAQ.

Resultados. Participaron 191 pacientes. ElTRAQ 5.0 pudo ser comprendido y completado por la mayoría de los pacientes $(96,3 \%)$, en forma autoadministrada, en poco tiempo (mediana: 5 minutos) y con poca o ninguna ayuda ( $81 \%)$. Presentar pobreza o escolaridad no acorde aumentó la necesidad de ayuda. La consistencia interna (alfa deCronbach) para la puntuación total fue 0,81 . Se demostró validez de construcción al testear diferentes hipótesis (todas $p<0,05$ ): discriminación según edad $\geq 16$ años (3,01 vs. 3,34 ), sexo (mujeres: $3,38>$ varones: 3,12 ) y presencia de proyecto futuro (sin: $3,01<\operatorname{con}: 3,34$ ); correlación con escala de autopercepción (r: 0,49). Conclusión. El TRAQ 5.0 queda disponible para ser utilizado en adolescentes argentinos con enfermedades crónicas.

Palabras clave: transición de la salud, estudios de validación, salud del adulto, enfermedad crónica, encuestas y cuestionarios.

http://dx.doi.org/10.5546/aap.2017.18

Texto completo en inglés:

http://dx.doi.org/10.5546/aap.2017.eng.18

\section{INTRODUCCIÓN}

Los avances en la medicina en las últimas décadas han aumentado la supervivencia de pacientes con enfermedades pediátricas crónicas complejas que llegan a la adultez.

Estos niños presentan necesidades especiales de atención de su salud, que, en la mayoría, continúan a medida que van creciendo y se hacen adultos. ${ }^{1}$

El pasaje de estos pacientes al seguimiento por profesionales de adultos es una realidad creciente que debe ser jerarquizada por el equipo de salud y entendida como un proceso y no un evento aislado. ${ }^{2}$ Es importante marcar la diferencia entre transferencia, entendida como el evento puntual del pasaje de una institución o equipo a otra, y transición, conceptualizada como un proceso dinámico, complejo y planificado, que incluye la transferencia propiamente dicha.

Los procesos de transición pueden ser especialmente críticos para la evolución de la enfermedad. Algunos pacientes abandonan los controles o tratamientos médicos al dejar la atención pediátrica, lo que constituye una situación de riesgo. ${ }^{3}$

Dada la complejidad de diversas enfermedades, se requiere que los adolescentes adquieran conocimientos y habilidades que permitan la continuidad de su cuidado. Debe ser gradual, pues implica un proceso planeado para la detección y puesta en marcha de recursos educacionales, médicos y de atención de los servicios de adultos. ${ }^{4,5}$ 
Desde la bibliografía, se propone administrar instrumentos en formato de cuestionarios para evaluar la autonomía de los pacientes al inicio del proceso y en etapas sucesivas, y definir progresos o dificultades para trabajar. ${ }^{5-7}$

Hasta la actualidad, no existía ningún instrumento validado en Argentina. De una revisión bibliográfica, aparecen disponibles diferentes instrumentos, algunos diseñados para ciertas patologías y otros genéricos, que pueden ser utilizados por pacientes con distintas enfermedades crónicas. ${ }^{8} \mathrm{El}$ instrumento genérico que por sus características aparece como más adecuado es el Transition Readiness Assessment Questionnaire (TRAQ). Este busca medir el grado de preparación para la transición de jóvenes con condiciones especiales de salud. Fue desarrollado por investigadores de la Universidad de Florida y validado para ser utilizado en adolescentes a partir de los 14 años. En su última versión original, el TRAQ 5.0, incluye 20 ítems y su validación mostró buenas propiedades psicométricas. ${ }^{9,10}$

El objetivo del presente estudio fue llevar a cabo la validación del instrumento TRAQ 5.0, versión en español argentino, en adolescentes y adultos jóvenes con enfermedades crónicas.

\section{POBLACIÓN, MATERIAL Y MÉTODOS}

\section{POBLACIÓN}

La población incluida comprendió pacientes atendidos en los siguientes servicios del Hospital Nacional de Pediatría Garrahan, en alguno de sus consultorios de seguimiento (si corresponde, se aclaran entre paréntesis): Crecimiento y Desarrollo, Gastroenterología (Enfermedades Inflamatorias Intestinales y Hepatología), Mielomeningocele, Neumonología (Asma, Enfermedad Pulmonar Obstructiva Crónica, Fibrosis Quística del Páncreas, Trasplante Pulmonar), Neurología (Enfermedad Neuromuscular y Epilepsia), Nutrición (Diabetes), Oncología (Tumores Sólidos y de Sistema Nervioso Central), Trasplantes Hepático y de Médula Ósea.

Se incluyeron, en una primera etapa, pacientes de 16 años o más que tuvieran aprobado, como mínimo, quinto grado a nivel escolar, entre julio de 2014 y junio de 2015. En una segunda etapa, al igual que para el instrumento original, se extendió la validación a pacientes de entre 14 años y 15 años y 11 meses, desde agosto de 2015 a noviembre de 2015. ${ }^{10}$

El reclutamiento fue de manera consecutiva y por conveniencia, y se excluyeron aquellos pacientes que ya habían tenido algún contacto para el seguimiento con un médico de adultos.

Se solicitó consentimiento informado a los pacientes y a sus padres o familiares. El estudio fue aprobado por los Comités de Ética y de Docencia e Investigación del Hospital.

\section{INSTRUMENTOS}

Transition Readiness Assessment Questionnaire 5.0, versión en español argentino

El TRAQ 5.0 incluye 20 ítems divididos en 5 subescalas: A. Manejo de medicación; B. Asistencia a citas; C. Seguimiento de los problemas de salud; D. Comunicación con los profesionales; y E. Manejo de las actividades cotidianas. Cada ítem se puntúa con una escala Likert de 1 a $5 ; 1$ equivale a mínima autonomía y 5, a máxima. La puntuación total y de las subescalas corresponde a la puntuación promedio entre los ítems respondidos. Se completa en forma autoadministrada (Anexo 1 en formato electrónico).

El proceso de adaptación transcultural al español argentino fue realizado por un equipo investigador del Hospital Italiano de Buenos Aires, y su última versión, aprobada por los autores del instrumento original (artículo en proceso de publicación). La última etapa de la adaptación transcultural, en la que se evaluó la comprensión de la versión local en una prueba en terreno, así como el proceso de validación que se muestra en este artículo, fueron realizados incluyendo a pacientes del Hospital Garrahan y por un equipo de trabajo conformado con investigadores de ambos hospitales.

\section{Cuestionario complementario completado por los pacientes}

Encuesta de opinión en la que se preguntó a los participantes sobre su experiencia al completar el TRAQ.

Para evaluar la proyección a futuro, se incluyeron también dos preguntas: “¿Cómo te ves en 5 años? ¿Y en 10 años?".

\section{Escalas analógico-visuales}

En la Figura 1, pueden observarse las escalas analógico-visuales (EAV) administradas a los médicos y a sus pacientes luego de completar el TRAQ. Para evaluar el grado de compromiso de la salud, se solicitó a los médicos de cabecera que tomaran en cuenta la gravedad y/o actividad actual de la enfermedad, según correspondiera, y la llevaran a una escala de 0 a 10 (de "sin 
compromiso" a "máximo compromiso"). Para contar con otra evaluación del nivel de preparación del paciente, se le solicitó que expresara su autopercepción en una escala global de 0 a 10 (de "nada" a "totalmente preparado").

\section{Cuestionario complementario completado por el investigador}

El investigador a cargo de las entrevistas registró variables sociodemográficas, clínicas y relacionadas con la administración del TRAQ.

Los datos aportados por los padres fueron edad, sexo, residencia, escolaridad de los pacientes y sus padres, cobertura y subsidio en salud, actividad actual del paciente y nivel socioeconómico evaluado por necesidades básicas insatisfechas (NBI), método directo para la medición de la pobreza estructural. Esta se determina por la presencia de, al menos, una de las siguientes condiciones: hacinamiento (más de 3 habitantes/cuarto), asistencia escolar (algún niño menor de 6-12 años sin escolaridad), vivienda y condiciones sanitarias (inquilinato, vivienda precaria, sin retrete) y capacidad de subsistencia (4 o más personas por miembro ocupado, con no más de $2^{\text {do }}$ grado). ${ }^{11}$ Se consideró con actividad actual a aquel paciente que trabajaba, estudiaba o tenía un familiar a cargo.

Los datos médicos se extrajeron de las historias clínicas y/o de los médicos de cabecera: diagnóstico, comorbilidades, órganos comprometidos, equipo principal que realizaba el seguimiento, especialidades que participaban del seguimiento, tiempo de evolución de la enfermedad, tratamiento actual y número de controles, visitas a la Guardia e internaciones en el último año.

Para evaluar la factibilidad en la administración del TRAQ, el investigador registró tiempo y posibilidad de llenado, necesidad de ayuda (mínima: menos de 4 veces; significativa: $\geq 4$ veces), forma de administración, dificultades con el formato y con algún ítem.

\section{Diseño}

Estudio descriptivo, transversal y cuantitativo. La administración del instrumento estuvo a cargo de una de las médicas investigadoras (GF), no relacionada con el seguimiento de los pacientes.

La validez de construcción fue analizada mediante el testeo de diferentes hipótesis, en las que se evaluaron los cambios en la puntuación del TRAQ según edad, sexo, gravedad de la enfermedad, presencia de un proyecto a futuro, y en relación con otra escala.

También se exploró la factibilidad y la validez de discriminación del TRAQ según nivel socioeconómico, cobertura en salud, nivel de instrucción y grupo de seguimiento conforme a la enfermedad.

\section{Estrategia}

De acuerdo con la organización de cada consultorio, se chequeó la asistencia de algún paciente de 14 años o más; se consultó la posibilidad de inclusión con los médicos de cabecera y se invitó a participar a los pacientes, generalmente en la sala de espera. Se solicitó a los pacientes que completaran el TRAQ en forma autoadministrada y con la posibilidad de pedir ayuda al investigador, la encuesta de opinión y la EAV de autopercepción global de preparación

FIGURA 1. Escalas analógico-visuales administradas a los pacientes y sus médicos

EAV de grado de compromiso de la enfermedad (respondida por su médico de cabecera)

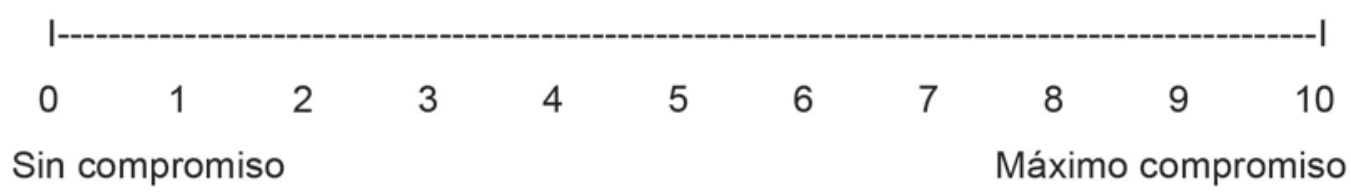

EAV de grado de preparación para la transición (respondida por el paciente)

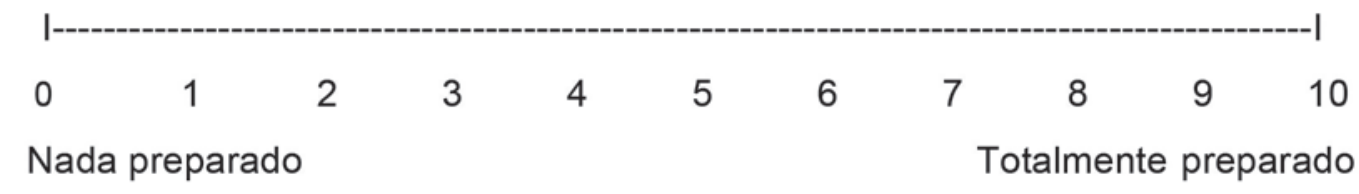


para el cuidado de su salud. En paralelo, se registraron datos sociodemográficos, médicos y sobre la administración del TRAQ. Por último, se solicitó al médico que completara la EAV correspondiente al compromiso actual de la enfermedad del paciente.

\section{Análisis estadístico}

Para evaluar la factibilidad de la administración, se estableció a priori la condición de que, al menos, un $80 \%$ de los participantes pudieran completar el instrumento. Los cuestionarios se consideraron como perdidos si el entrevistado no era capaz de responder más del $50 \%$ de los ítems, a pesar de recibir ayuda. Se describieron las dificultades, mediana de tiempo y ayuda necesaria para su administración y su asociación con el nivel de instrucción (considerando escolaridad no acorde a la edad como 2 años o más de diferencia con respecto a lo que correspondería por edad o escuela especial) y pobreza (NBI), a través del test de Fisher o chi cuadrado.

Se describió la distribución de las puntuaciones a través de las medias, desvío estándar (DE), rangos y magnitud del efecto piso y techo para cada puntuación/dominio. Se consideró que había efecto piso o techo si más del 15\% de los participantes habían utilizado los valores extremos. ${ }^{12}$

La confiabilidad de la escala se evaluó con la consistencia interna del instrumento mediante el coeficiente $\alpha$ de Cronbach para la puntuación total y cada subescala, tomando como límite, para la comparación de grupos, un valor de 0,70 y, para análisis individual, de 0,90.

Las respuestas otorgadas por los pacientes en relación con su proyección a futuro fueron analizadas por las 3 investigadoras principales $y$, según su contenido, dicotomizadas en con o sin proyecto futuro.

La validez de discriminación se estudió a partir de diferencias en las puntuaciones entre grupos conocidos: sexo, actividad actual del paciente y percepción a futuro. También se realizó un análisis exploratorio de presencia de diferencias por NBI, nivel de instrucción y grupo de seguimiento. Se utilizó la prueba de T para muestras independientes o análisis de varianza (analysis of variance; ANOVA, por sus siglas en inglés) para variables dicotómicas o categóricas, respectivamente.

La búsqueda de correlación entre los resultados del TRAQ y la edad, el compromiso de enfermedad y la percepción de preparación para la transición se realizó mediante el test de Pearson.

Para los análisis, un valor de $\mathrm{p}<0,05$ fue considerado como significativo. Se utilizó, para procesar los datos, el programa estadístico SPSS11.5.

Los resultados se reportaron globalmente, solo diferenciando los grupos por edad (14-16 años y mayores de 16 años) si se encontraron diferencias significativas.

\section{RESULTADOS}

Para la primera etapa, de los 142 pacientes invitados a participar, finalmente, 2 no fueron incluidos, uno por falta de tiempo y otro por negativa posterior. En la segunda etapa, de 54 pacientes invitados, 3 no tuvieron deseo de participar. En total, se incluyeron 191 pacientes.

En la Tabla 1, se muestran las características sociodemográficas. La distribución por sexos fue homogénea. La edad media fue de 16,9 años (DE: 1,57), con amplio rango etario, que alcanzó los 26 años. La mayoría provenía del interior del país. Casi la mitad tenían certificado de discapacidad, y un cuarto presentaba escolaridad no acorde.

\section{Factibilidad}

Los resultados de la evaluación de la factibilidad del uso del TRAQ 5.0 se muestran en la Tabla 2.

En general, el instrumento fue bien comprendido y pudo ser completado en poco tiempo. No hubo grandes dificultades con el formato.

Solo $7(3,7 \%)$ no pudieron completarlo. De ellos, tres tenían diagnóstico de discapacidad intelectual; dos, por su patología de base, tenían escuela domiciliaria prolongada; uno tenía maestra integradora; y el otro había abandonado la escuela hacía más de 5 años.

De los 184 pacientes que sí pudieron completarlo, el $81 \%$ pudo hacerlo sin ayuda. Del 19\% que requirió ayuda, solo 3 pacientes, significativa. Se observó que tanto la presencia de NBI como la escolaridad no acorde aumentaban la necesidad de ayuda: con NBI, $36,4 \%$ vs. $\sin$ NBI, $16,7 \%$, p: 0,027; con escolaridad no acorde, $36,6 \%$ vs. acorde, $13,9 \%$, p: 0,001.

La mayoría de los pacientes respondió en forma autoadministrada. Solo en 12 casos, fue leída por el investigador: 8 por debilidad muscular, 3 por alteraciones visuales y uno por 
dificultad en la lectura.

Según la opinión de los pacientes, a la mayoría le gustó responder el instrumento $(67,9 \%)$, y las preguntas resultaron fáciles $(62 \%)$ e importantes $(79,9 \%)$. Al 38\% le pareció que le habían preguntado algo nuevo, que no habían pensado antes.

\section{Distribución de las puntuaciones}

En la Tabla 3, se describe la distribución de las puntuaciones. Todos los pacientes utilizaron el rango completo de las opciones para los 20 ítems. Excepto para los dominios de comunicación con los profesionales y el manejo de actividades cotidianas, en los que el efecto techo fue moderado, el efecto piso y techo fue nulo o mínimo en el resto

TABLA 1. Características sociodemográficas de la población incluida en la validación del Transition Readiness Assessment Questionnaire 5.0, versión en español argentino

\begin{tabular}{|c|c|c|}
\hline & $\mathbf{N}$ & Media (DE)/\% \\
\hline Edad & 191 & $16,9(1,57)$ \\
\hline \multicolumn{3}{|l|}{ Sexo } \\
\hline$\square$ Mujeres & 100 & $52,4 \%$ \\
\hline$\square$ Varones & 91 & $47,6 \%$ \\
\hline \multicolumn{3}{|l|}{ Lugar de residencia } \\
\hline$\square$ Provincia de Buenos Aires & 114 & $59,7 \%$ \\
\hline$\square \quad$ Ciudad Autónoma de Buenos Aires & 14 & $7,3 \%$ \\
\hline$\square \quad$ Resto del interior del país & 60 & $31,4 \%$ \\
\hline$\square$ Paraguay & 3 & $1,6 \%$ \\
\hline Necesidades básicas insatisfechas & 24 & $12,6 \%$ \\
\hline Sin cobertura en salud & 55 & $28,8 \%$ \\
\hline \multicolumn{3}{|l|}{ Subsidio en salud } \\
\hline$\square \quad$ Ninguno & 78 & $40,8 \%$ \\
\hline $\begin{array}{l}\square \text { Pensión por discapacidad (incluye certificado de } \\
\text { discapacidad) }\end{array}$ & 82 & $42,9 \%$ \\
\hline$\square \quad$ Certificado de discapacidad & 31 & $16,2 \%$ \\
\hline \multicolumn{3}{|l|}{ Nivel de educación ${ }^{1}$} \\
\hline$\square \quad$ Acorde a su edad & 145 & $75,9 \%$ \\
\hline$\square \quad$ No acorde a su edad & 46 & $24,1 \%$ \\
\hline \multicolumn{3}{|l|}{ Tipo de educación } \\
\hline$\square$ Común & 174 & $91,1 \%$ \\
\hline$\square \quad$ Común con integración & 10 & $5,2 \%$ \\
\hline$\square$ Domiciliaria & 4 & $2,1 \%$ \\
\hline$\square \quad$ Especial por discapacidad intelectual & 2 & $1 \%$ \\
\hline$\square \quad$ Especial por compromiso motor & 1 & $0,5 \%$ \\
\hline
\end{tabular}

1. Nivel de educación: se establece un límite de 2 años o más de diferencia con respecto a lo que le correspondería por edad o escuela especial.

DE: desvío estándar. 
de los dominios y para la puntuación total. Esta distribución asegura que las opciones ofrecidas por el instrumento abarcan el espectro de opciones requeridas para la población.

\section{Confiabilidad}

Puede observarse, en la Tabla 3, que el coeficiente de confiabilidad $\alpha$ de Cronbach para la puntuación total fue de 0,81 . Sin embargo, la confiabilidad de las subescalas no alcanza el límite recomendado de 0,7, excepto en Asistencia a citas, lo que determina que solo debe utilizarse el instrumento para realizar comparaciones a través de su puntuación total.

\section{Validez}

La validez de construcción y discriminante del instrumento se comprobó a partir de las hipótesis planteadas (Tabla 4):

1. Hubo correlación positiva entre edad y puntuación total del TRAQ (r: 0,286; $\mathrm{p}:<0,001)$, y diferencias al comparar las puntuaciones por grupo etario.

2. Las mujeres presentaron mejores puntuaciones que los varones.

3. Existió correlación negativa entre la puntuación total y el grado de compromiso de enfermedad (EAV) solo en el grupo de mayores de 16 años, pero sin alcanzar significancia estadística.

4. La autopercepción global de la preparación de los pacientes (EAV) correlacionó positivamente con la puntuación total.

5. Aquellos pacientes que tenían proyección a futuro o una percepción positiva de este presentaron mejores puntuaciones que aquellos que no. Las diferencias no fueron significativas en el grupo de 14-16 años.

Por último, al explorar si los resultados del TRAQ discriminaban por NBI, cobertura en salud, escolaridad acorde a edad y grupo de reclutamiento, solo se encontraron diferencias en esta última variable.

En la Tabla 5, puede observarse la relación entre las puntuaciones y los diferentes grupos de reclutamiento, y se encuentran diferencias significativas en la puntuación total y los dominios de medicación, asistencia a citas y manejo de actividades cotidianas. El grupo de Neurología aparece como diferente, con autorreferencia de menor preparación.

\section{DISCUSIÓN}

El instrumento TRAQ 5.0, versión en español -argentino, resultó comprensible y factible de ser utilizado en la población de adolescentes-adultos jóvenes con enfermedades crónicas seguidos en un hospital de alta complejidad a partir de los 14 años. Fue fácil de administrar y demandó escaso tiempo.

Si bien la mayoría no requirió ayuda para completar el cuestionario, quienes sí

Tabla 2. Administración del Transition Readiness Assessment Questionnaire 5.0 en español argentino: dificultades, necesidad de ayuda y tiempo en adolescentes/adultos jóvenes. N: 191

\begin{tabular}{|c|c|c|}
\hline \multicolumn{2}{|c|}{ Aspecto evaluado } & \\
\hline \multicolumn{2}{|l|}{ Pudo completarlo } & $96,3 \%$ \\
\hline \multirow{3}{*}{ Ayuda requerida $^{1}$} & Sin ayuda & $81 \%$ \\
\hline & Mínima $^{2}$ & $17,4 \%$ \\
\hline & Significativa ${ }^{2}$ & $1,6 \%$ \\
\hline \multirow[t]{2}{*}{ Forma de administración ${ }^{1}$} & Adecuada & $93,5 \%$ \\
\hline & Leído por el investigador & $6,5 \%$ \\
\hline \multirow{3}{*}{$\begin{array}{l}\text { Dificultades con el } \\
\text { formato }^{1}\end{array}$} & Sin dificultades & $83,7 \%$ \\
\hline & Por olvido & $7,6 \%$ \\
\hline & Por superposición & $8,7 \%$ \\
\hline \multicolumn{2}{|c|}{ Tiempo de llenado $^{1}$ (mediana) } & 5 minutos $(3-20)$ \\
\hline
\end{tabular}

1. Solo se consideró para estos cálculos al grupo que sí pudo completar el instrumento (n: 184).

2. Se consideró ayuda mínima: $<4$ veces; significativa: $\geq 4$ veces. 
la necesitaron, en su mayoría, presentaban como grupo más antecedentes de discapacidad intelectual, escolaridad no acorde o pertenecían a familias pobres. Esta necesidad de ayuda ya había sido constatada en un estudio previo de validación de un instrumento para evaluar calidad de vida (PedsQL 4.0) en una población similar del mismo hospital. ${ }^{13}$

En relación con las propiedades psicométricas, la consistencia interna del instrumento mostró ser satisfactoria para la puntuación total con el coeficiente $\alpha$ de Cronbach, que excedió el límite recomendado para la comparación de grupos $(0,70)$. Comparando con la última versión publicada en 2014 por los autores del instrumento, los coeficientes $\alpha$ de Cronbach en nuestra muestra fueron menores tanto para la puntuación total $(0,94$ vs. 0,81$)$ como para los diferentes dominios por separado. ${ }^{10}$

Mediante el tratamiento de las hipótesis planteadas a priori, se pudo probar que el instrumento era válido para la población global. Aquellas en referencia al sexo y la edad habían sido también evaluadas en la validación del cuestionario original y sus resultados coincidentes.

A pesar de que los autores del artículo original habían testeado la validez de construcción buscando relación con la etnia de los pacientes y la presencia de cobertura médica, en la

Tabla 3. Descripción de la distribución de las puntuaciones y confiabilidad en el Transition Readiness Assessment Questionnaire 5.0, versión en español argentino $(n=184)$

\begin{tabular}{|c|c|c|c|c|c|c|c|c|}
\hline \multirow{2}{*}{ Puntuación/dominio } & \multirow{2}{*}{ Media } & \multirow{2}{*}{$\mathrm{DE}$} & \multirow{2}{*}{ Rango } & \multirow{2}{*}{$\begin{array}{c}\text { Efecto } \\
\text { piso } \\
\%\end{array}$} & \multirow{2}{*}{$\begin{array}{c}\text { Efecto } \\
\text { techo } \\
\%\end{array}$} & \multicolumn{3}{|c|}{$\begin{array}{l}\text { Coeficiente } \alpha \\
\text { de Cronbach }\end{array}$} \\
\hline & & & & & & $\begin{array}{c}\text { De } 14 \text { a } 15 \\
\text { años y } 11 \\
\text { meses } \\
(n=49)\end{array}$ & $\begin{array}{l}\geq 16 \\
\text { años } \\
(n= \\
135)\end{array}$ & $\begin{array}{l}\text { Total } \\
(\mathrm{n}= \\
184)\end{array}$ \\
\hline Total & 3,26 & 0,64 & $\begin{array}{l}1,1- \\
4,70\end{array}$ & 0,5 & 0,5 & 0,81 & 0,80 & 0,81 \\
\hline $\begin{array}{l}\text { Manejo de la } \\
\text { medicación }\end{array}$ & 3,63 & 0,91 & $1-5$ & 1,1 & 6,7 & 0,68 & 0,58 & 0,61 \\
\hline Asistencia a citas & 2,63 & 0,81 & $1-4,71$ & 2,7 & 0,5 & 0,62 & 0,71 & 0,71 \\
\hline $\begin{array}{l}\text { Seguimiento de los } \\
\text { problemas de salud }\end{array}$ & 2,96 & 0,89 & $\begin{array}{l}1,25- \\
4,75\end{array}$ & 2,7 & 1,1 & 0,38 & 0,25 & 0,30 \\
\hline $\begin{array}{l}\text { Comunicación con } \\
\text { los profesionales }\end{array}$ & 4,19 & 0,98 & $1-5$ & 2,2 & 41,3 & 0,45 & 0,54 & 0,52 \\
\hline $\begin{array}{l}\text { Manejo de las } \\
\text { actividades } \\
\text { cotidianas }\end{array}$ & 4,03 & 0,93 & $1-5$ & 1,6 & 23,9 & 0,63 & 0,51 & 0,55 \\
\hline
\end{tabular}

Nota: Se consideró que había efecto piso o techo si era mayor del 15\%. La magnitud de estos se designa como mínimo $(0,20)$, moderado $(0,50)$ y grande $(0,80)$.

DE: desvío estándar. 
Tabla 4. Validez de construcción del Transition Readiness Assessment Questionnaire 5.0, versión en español argentino

\begin{tabular}{|c|c|c|c|c|c|c|c|c|c|c|}
\hline & \multicolumn{3}{|c|}{ 14-15 años } & \multicolumn{4}{|c|}{$\geq 16$ años } & \multicolumn{3}{|c|}{$\mathrm{p}^{*}$} \\
\hline $\begin{array}{l}\text { Puntuación total } \\
\text { TRAQ }\end{array}$ & \multicolumn{3}{|c|}{$3,01 \pm 0,65$} & \multicolumn{4}{|c|}{$3,34 \pm 0,61$} & \multicolumn{3}{|c|}{0,002} \\
\hline \multicolumn{11}{|c|}{$2^{a}$ hipótesis: "Las mujeres presentarán mejores resultados que los varones". } \\
\hline & $\begin{array}{c}\text { Puntuación } \\
\text { total } \\
\text { 14-15 años }\end{array}$ & \multicolumn{2}{|l|}{$\mathrm{p}^{*}$} & \multicolumn{2}{|c|}{$\begin{array}{l}\text { Puntuación } \\
\text { total } \\
\geq 16 \text { años }\end{array}$} & \multicolumn{2}{|c|}{$\mathrm{p}^{*}$} & \multicolumn{2}{|c|}{$\begin{array}{l}\text { Puntuación } \\
\text { total global }\end{array}$} & $\mathrm{p}^{*}$ \\
\hline Mujeres & $3,21 \pm 0,58$ & \multirow{2}{*}{\multicolumn{2}{|c|}{0,023}} & \multirow{2}{*}{\multicolumn{2}{|c|}{$\begin{array}{l}3,44 \pm 0,55 \\
3,24 \pm 0,66\end{array}$}} & \multirow{2}{*}{\multicolumn{2}{|c|}{0,057}} & \multirow{2}{*}{\multicolumn{2}{|c|}{\begin{tabular}{|l|}
$3,38 \pm 0,57$ \\
$3,12 \pm 0,69$ \\
\end{tabular}}} & \multirow[t]{2}{*}{0,006} \\
\hline Varones & $2,79 \pm 0,67$ & & & & & & & & & \\
\hline \multicolumn{11}{|c|}{$\begin{array}{l}3^{a} \text { hipótesis: "Aquellos pacientes con mayor gravedad o nivel de actividad actual en su } \\
\text { enfermedad crónica presentarán puntuaciones más bajas". }\end{array}$} \\
\hline & \multicolumn{3}{|c|}{\begin{tabular}{|c|} 
Puntuación total \\
TRAQ 5.0 \\
Media (DE)
\end{tabular}} & \multicolumn{2}{|c|}{\begin{tabular}{c|} 
EAV del \\
médico \\
Media (DE)
\end{tabular}} & \multicolumn{3}{|c|}{$r$} & \multicolumn{2}{|c|}{$\mathrm{p}^{\star *}$} \\
\hline $14-15$ años & \multicolumn{2}{|c|}{$3,01(0,65)$} & \multicolumn{3}{|c|}{$4,80(2,62)$} & \multicolumn{3}{|c|}{0,064} & \multicolumn{2}{|c|}{0,67} \\
\hline$\geq 16$ años & \multicolumn{2}{|c|}{$3,34(0,61)$} & \multicolumn{3}{|c|}{$4,77(2,79)$} & \multicolumn{3}{|c|}{$-0,17$} & \multicolumn{2}{|c|}{0,054} \\
\hline Global & \multicolumn{2}{|c|}{$3,26(0,64)$} & \multicolumn{3}{|c|}{$4,78(2,74)$} & \multicolumn{3}{|c|}{$-0,107$} & \multicolumn{2}{|c|}{0,153} \\
\hline $\begin{array}{l}4^{a} \text { hipótesis: "E } \\
\text { transferencia del }\end{array}$ & $\begin{array}{l}\text { tirá concord } \\
\text { spio paciente }\end{array}$ & cia $\epsilon$ & & $\begin{array}{l}\text { e la perce } \\
\text { oor una } E A\end{array}$ & & & res & & $\begin{array}{l}\text { ción } \\
\text { el TF }\end{array}$ & ara la \\
\hline & $\begin{array}{l}\text { Puntuación } \\
\text { total } \\
\text { TRAQ 5.0 } \\
\text { Media (DE) }\end{array}$ & & & $\begin{array}{l}\text { V del } \\
\text { ciente } \\
\text { ia (DE) }\end{array}$ & & & $r$ & & $p^{*}$ & \\
\hline 14-15 años & $3,01(0,65)$ & &, 18 & $(3,24)$ & & & 32 & & 0,0 & \\
\hline$\geq 16$ años & $3,34(0,61)$ & &, 18 & $(2,45)$ & & & 52 & & $<0$, & 01 \\
\hline Global & $3,26(0,64)$ & & 65 & $(2,81)$ & & & 49 & & $<0$, & 01 \\
\hline $\begin{array}{l}5^{a} \text { hipótesis: "A } \\
\text { ocupación o tuvie }\end{array}$ & $\begin{array}{l}\text { Iellos pacient } \\
\text { in un proyecto }\end{array}$ & $\begin{array}{l}\text { s que } \\
\text { futuro }\end{array}$ & & $\begin{array}{l}\text { encuentr } \\
\text { drán puntua }\end{array}$ & ren & & liza & & vamen & e una \\
\hline & $\begin{array}{l}\text { Puntuación } \\
\text { total } \\
14-15 \text { años }\end{array}$ & $\mathrm{p}^{*}$ & & $\begin{array}{l}\text { Intuación } \\
\text { total } \\
16 \text { años }\end{array}$ & $\mathrm{p}^{*}$ & & & $\begin{array}{l}\text { tua } \\
\text { glc }\end{array}$ & total & $\mathrm{p}^{*}$ \\
\hline $\begin{array}{l}\text { Con } \\
\text { plan/proyecto a } \\
\text { futuro (5 años) }\end{array}$ & $3,19 \pm 0,72$ & 0,49 & & $49 \pm 0,55$ & 0,0 & & & 42 &, 60 & 0,004 \\
\hline $\begin{array}{l}\text { Sin } \\
\text { plan/proyecto a } \\
\text { futuro (5 años) }\end{array}$ & $3,03 \pm 0,53$ & & & $17 \pm 0,64$ & & & & 14 & 62 & \\
\hline $\begin{array}{c}\text { Con } \\
\text { plan/proyecto a } \\
\text { futuro (10 años) }\end{array}$ & $3,23 \pm 0,76$ & 0,44 & & $48 \pm 0,52$ & 0,02 & & & 43 &, 58 & 0,019 \\
\hline $\begin{array}{c}\text { Sin } \\
\text { plan/proyecto a } \\
\text { futuro (10 años) }\end{array}$ & $3,05 \pm 0,57$ & & & $25 \pm 0,66$ & & & & 20 &, 64 & \\
\hline
\end{tabular}

* Prueba de T. ** Análisis de varianzas (ANOVA).

TRAQ: Transition Readiness Assessment Questionnaire; DE: desvío estándar; EAV: escalas analógico-visuales. 
experiencia local, se decidió no considerar estas dos variables por no encontrarse estas diferencias con frecuencia en nuestra población.

Además, en la presente experiencia, se testearon los resultados del TRAQ utilizando dos nuevas hipótesis. La primera analizó la correlación entre las puntuaciones del TRAQ y una medición externa global de la autopercepción de preparación (EAV), que fortaleció la validez de este instrumento. ${ }^{13} \mathrm{La}$ otra fue la búsqueda de concordancia entre el compromiso de la salud y los resultados del TRAQ. La falta de correlación en este estudio debe ser analizada en futuras investigaciones, ya que fue una hipótesis exploratoria no utilizada previamente.

Debe destacarse que una limitación de este estudio es que fue validado con la población de un solo hospital y en un número menor de pacientes, a diferencia del estudio original, en el que los pacientes provinieron de 3 diferentes centros. Sin embargo, el Hospital Garrahan atiende a una población heterogénea de pacientes y se considera la posibilidad de incorporar pacientes atendidos en el Hospital Italiano en una segunda etapa. Queda pendiente la evaluación de la sensibilidad al cambio que presenta el instrumento, útil para ser utilizado en estudios longitudinales para evaluar intervenciones.

En resumen, el TRAQ 5.0 queda disponible para evaluar el nivel de preparación de pacientes argentinos con enfermedades crónicas a partir de los 14 años. Estudios posteriores permitirán conocer su utilidad en el proceso de transición a la medicina del adulto de los adolescentes con enfermedades crónicas.

\section{Agradecimientos}

Agradecemos la participación de los pacientes y sus familias, así como de los profesionales que trabajan en los distintos sectores del hospital donde se llevó a cabo el estudio.

TABla 5. Descripción de los resultados del Transition Readiness Assessment Questionnaire 5.0 según el lugar de reclutamiento

\begin{tabular}{|c|c|c|c|c|c|c|c|c|c|c|c|}
\hline \multirow[t]{2}{*}{$\begin{array}{l}\text { Puntuación/ } \\
\text { Dominio }\end{array}$} & $\begin{array}{l}C y D \\
(n: 24)\end{array}$ & $\begin{array}{r}T M O \\
(n: 11)\end{array}$ & $\begin{array}{l}\text { Neumo. } \\
\text { (n:34) }\end{array}$ & $\begin{array}{l}\text { Onco. } \\
\text { (n: 26) }\end{array}$ & $\begin{array}{r}M M C \\
\text { (n: 19) }\end{array}$ & $\begin{array}{r}D B T \\
(n: 20)\end{array}$ & $\begin{array}{l}\text { Gastro. } \\
\text { (n: 23) }\end{array}$ & $\begin{array}{l}\text { Neuro. } \\
\text { (n: 21) }\end{array}$ & $\begin{array}{c}\text { Tx. } \\
\text { Hepático } \\
\text { (n: 20) }\end{array}$ & \multirow[t]{2}{*}{$f$} & \multirow[t]{2}{*}{$\mathrm{P}^{*}$} \\
\hline & $\begin{array}{l}\text { Media } \\
\pm D E\end{array}$ & $\begin{array}{l}\text { Media } \\
\pm D E\end{array}$ & $\begin{array}{l}\text { Media } \\
\pm D E\end{array}$ & $\begin{array}{l}\text { Media } \\
\pm D E\end{array}$ & $\begin{array}{l}\text { Media } \\
\pm D E\end{array}$ & $\begin{array}{l}\text { Media } \\
\pm D E\end{array}$ & $\begin{array}{l}\text { Media } \pm \\
\text { DE }\end{array}$ & $\begin{array}{l}\text { Media } \\
\pm D E\end{array}$ & $\begin{array}{l}\text { Media } \pm \\
\text { DE }\end{array}$ & & \\
\hline Total & $\begin{array}{l}3,16 \pm \\
0,59\end{array}$ & $\begin{array}{l}3,52 \pm \\
0,60\end{array}$ & $\begin{array}{c}3,12 \pm \\
0,54\end{array}$ & $\begin{array}{c}3,49 \pm \\
0,74\end{array}$ & $\begin{array}{c}2,94 \pm \\
0,63\end{array}$ & $\begin{array}{c}3,64 \pm \\
0,59\end{array}$ & $\begin{array}{c}3,26 \pm \\
0,51\end{array}$ & $\begin{array}{c}2,78 \pm \\
0,67\end{array}$ & $\begin{array}{c}3,35 \pm \\
0,64\end{array}$ & 3,85 & $<0,001^{1}$ \\
\hline Medicación & $\begin{array}{c}3,40 \pm \\
0,79\end{array}$ & $\begin{array}{c}3,88 \pm \\
1,02\end{array}$ & $\begin{array}{c}3,54 \pm \\
0,77\end{array}$ & $\begin{array}{c}4,04 \pm \\
0,99\end{array}$ & $\begin{array}{c}3,19 \pm \\
1,04\end{array}$ & $\begin{array}{c}4,00 \pm \\
0,70\end{array}$ & $\begin{array}{c}3,69 \pm \\
0,77\end{array}$ & $\begin{array}{c}3,10 \pm \\
1,09\end{array}$ & $\begin{array}{c}3,56 \pm \\
0,93\end{array}$ & 2,48 & $0,015^{2}$ \\
\hline $\begin{array}{c}\text { Asistencia } \\
\text { a citas }\end{array}$ & $\begin{array}{c}2,56 \pm \\
0,66\end{array}$ & $\begin{array}{c}2,94 \pm \\
0,77\end{array}$ & $\begin{array}{c}2,50 \pm \\
0,76\end{array}$ & $\begin{array}{c}2,92 \pm \\
0,87\end{array}$ & $\begin{array}{c}2,17 \pm \\
0,83\end{array}$ & $\begin{array}{c}2,95 \pm \\
0,82\end{array}$ & $\begin{array}{c}2,58 \pm \\
0,69\end{array}$ & $\begin{array}{c}2,12 \pm \\
0,78\end{array}$ & $\begin{array}{c}2,84 \pm \\
0,82\end{array}$ & 2,97 & $0,004^{3}$ \\
\hline $\begin{array}{l}\text { Seguimiento } \\
\text { de problemas } \\
\text { de salud }\end{array}$ & $\begin{array}{c}2,89 \pm \\
1,09\end{array}$ & $\begin{array}{c}3,31 \pm \\
0,84\end{array}$ & $\begin{array}{c}2,76 \pm \\
0,86\end{array}$ & $\begin{array}{c}3,04 \pm \\
0,88\end{array}$ & $\begin{array}{c}3,09 \pm \\
0,62\end{array}$ & $\begin{array}{c}3,37 \pm \\
0,95\end{array}$ & $\begin{array}{c}2,84 \pm \\
0,98\end{array}$ & $\begin{array}{c}2,57 \pm \\
0,80\end{array}$ & $\begin{array}{c}2,94 \pm \\
0,71\end{array}$ & 1,52 & 0,152 \\
\hline $\begin{array}{l}\text { Comunicación } \\
\text { con los } \\
\text { profesionales }\end{array}$ & $\begin{array}{c}4,27 \pm \\
0,80\end{array}$ & $\begin{array}{c}3,80 \pm \\
1,22\end{array}$ & $\begin{array}{c}4,07 \pm \\
1,12\end{array}$ & $\begin{array}{c}4,22 \pm \\
1,06\end{array}$ & $\begin{array}{c}3,90 \pm \\
1,24\end{array}$ & $\begin{array}{c}4,47 \pm \\
0,73\end{array}$ & $\begin{array}{c}4,24 \pm \\
0,83\end{array}$ & $\begin{array}{c}4,30 \pm \\
0,84\end{array}$ & $\begin{array}{c}4,31 \pm \\
0,90\end{array}$ & 0,76 & 0,63 \\
\hline $\begin{array}{l}\text { Manejo de las } \\
\text { actividades } \\
\text { cotidianas }\end{array}$ & $\begin{array}{c}4,03 \pm \\
0,80\end{array}$ & $\begin{array}{c}4,49 \pm \\
0,54\end{array}$ & $\begin{array}{c}3,85 \pm \\
0,89\end{array}$ & $\begin{array}{c}4,20 \pm \\
0,91\end{array}$ & $\begin{array}{c}3,56 \pm \\
0,87\end{array}$ & $\begin{array}{c}4,53 \pm \\
0,58\end{array}$ & $\begin{array}{c}4,19 \pm \\
0,71\end{array}$ & $\begin{array}{c}3,20 \pm \\
1,40\end{array}$ & $\begin{array}{c}4,14 \pm \\
0,92\end{array}$ & 3,97 & $<0,001^{4}$ \\
\hline
\end{tabular}

C y D: Crecimiento y Desarrollo; TMO: Trasplante de Médula Ósea; Neumo.: Neumonología; Onco.: Oncología; MMC: Mielomeningocele; DBT: Diabetes; Gastro.: Gastroenterología; Neuro.: Neurología; Tx. Hepático:

Trasplante Hepático.

* Análisis de varianzas (ANOVA). 1 Neuro. < DBT, TMO, oncológicos; 2 Neuro. < oncológicos, DBT, TMO; 3

Neuro. < DBT, TMO, oncológicos; 4 Neuro. < DBT, TMO, oncológicos, gastro., tx. hepático.

DE: desvío estándar. 


\section{REFERENCIAS}

1. McPherson M, Arango P, Fox H, Lauver C, et al. A new definition of Children with Special Health Care Needs. Pediatrics 1998;102(1 pt 1):137-40.

2. Jiménez Domínguez R, Cavalcante MTL. Proceso de transición de hospital pediátrico a hospital de adultos en pacientes crónicos complejos del Hospital Sant Joan de Déu. Documentos de Trabajo Social 2013;52:166-213.

3. Carpi JM. Transición a los cuidados del adulto. Enfermedad Inflamatoria Intestinal al día 2012;11(3):187-95.

4. Ferris ME, Harward DH, Bickford K, Layton JB, et al. A clinical tool to measure the components of health-care transition from pediatric care to adult care: The UNC TR(x) ANSITION Scale. Ren Fail 2012;34(6):744-53.

5. De Cunto CL. Transición en la atención médica, de la pediatría a la medicina del adulto. Arch Argent Pediatr 2012;110(4);341-7.

6. American Academy of Pediatrics, American Academy of Family Physicians, American College of PhysiciansAmerican Society of Internal Medicine. A consensus statement on health care transitions for young adults with special health care needs. Pediatrics 2002;110(6 pt 2):1304-6.

7. American Academy of Pediatrics, American Academy of Family Physicians, American College of Physicians, Transitions Clinical Report Authoring Group. Supporting the health care transition from adolescence to adulthood in the medical home. Pediatrics 2011;128 (1):182-200.

8. Zhang LF, Ho JS, Kennedy SE. A systematic review of the psychometric properties of transition readiness assessment tools in adolescents with chronic disease. BMC Pediatr 2014,14:4.

9. Sawicki GS, Lukens-Bull K, Yin X, Demars N, et al. Meassuring the transition readiness of youth with special healthcare needs: Validation of the TRAQ- Transition Readiness Assessment Questionnaire. J Pediatr Psychol 2011;36(2):160-71.

10. Wood DL,Sawicki GS, Miller MD,Smotherman Cetal. The Transition Readiness Assessment Questionnaire (TRAQ): its factor structure, reliability, and validity. Acad Pediatr 2014;14(4):415-22.

11. INDEC. La pobreza en la Argentina. Serie Estudios N. ${ }^{\circ} 1$. Buenos Aires: INDEC, 1984.

12. Terwee $C B$, Bot $S D$, de Boer MR, van der Windt DA, et al. Quality criteria were proposed for measurement properties of health status questionnaires. J Clin Epidemiol 2007;60(1):34-42.

13. Roizen M, Rodríguez S, Bauer G, Medin G, et al. Initial validation of the ArgentineanSpanish version of the PedsQL 4.0 Generic Core Scales in children and adolescents with chronic diseases: acceptability and comprehensibility in low-income settings. Health Qual Life Outcomes 2008;6:59. 


\section{ANEXO 1}

Nombre del paciente:

Fecha de nacimiento:

Fecha de hoy

\section{Cuestionario de Evaluación para la Preparación de la Transición (TRAQ)}

Instrucciones para los jóvenes: Por favor marcá con una cruz la opción que mejor describa tu capacidad para cada una de las siguientes áreas que son importantes para la transición del cuidado de tu salud a la medicina del adulto. No hay respuestas correctas ni incorrectas y las respuestas serán confidenciales y privadas.

Instrucciones para padres/cuidadores: si el joven que está a su cuidado no tiene la capacidad de comprender el significado por sí mismo de las preguntas que se mencionan más abajo, por favor, respóndalas en forma conjunta y marque con una cruz el casillero que mejor describa la capacidad del joven para realizarlas.

Marque con una cruz, si usted es (padres/cuidadores) quien está completando el formulario.

\begin{tabular}{|c|c|c|c|c|c|}
\hline & $\begin{array}{l}\text { No, no sé } \\
\text { cómo } \\
\text { hacerlo. }\end{array}$ & $\begin{array}{l}\text { No, pero } \\
\text { quiero } \\
\text { aprender a } \\
\text { hacerlo. }\end{array}$ & $\begin{array}{l}\text { No, pero estoy } \\
\text { aprendiendo a } \\
\text { hacerlo. }\end{array}$ & $\begin{array}{l}\text { Si, ya he } \\
\text { comenzado } \\
\text { a hacerlo. }\end{array}$ & $\begin{array}{l}\text { Si, lo hago } \\
\text { siempre } \\
\text { que lo } \\
\text { necesito. }\end{array}$ \\
\hline \multicolumn{6}{|l|}{$\begin{array}{l}\text { Manejo de la medicación (para pacientes que han tomado o toman } \\
\text { medicación) }\end{array}$} \\
\hline \multicolumn{6}{|l|}{ 1. ¿Si la necesitás, vas a buscar tu medicación a la farmacia? } \\
\hline \multicolumn{6}{|l|}{$\begin{array}{l}\text { 2. ¿Sabés qué hacer si tenés una reacción adversa a tu medicación } \\
\text { (por ejemplo: reacción alérgica, diarrea por la medicación)? }\end{array}$} \\
\hline \multicolumn{6}{|l|}{$\begin{array}{l}\text { 3. ¿Tomás por vos mismo tus medicamentos tal como te los } \\
\text { indicaron? }\end{array}$} \\
\hline \multicolumn{6}{|l|}{ 4. ¿Solicitás tus medicamentos antes de que se terminen? } \\
\hline \multicolumn{6}{|l|}{ Asistencia a citas } \\
\hline \multicolumn{6}{|l|}{$\begin{array}{l}\text { 5. ¿Te ocupás vos mismo de pedir los turnos para los consultorios } \\
\text { médicos? }\end{array}$} \\
\hline \multicolumn{6}{|l|}{$\begin{array}{l}\text { 6. ¿Estás pendiente de las derivaciones a especialistas, controles } 0 \\
\text { de los resultados de análisis de laboratorio? }\end{array}$} \\
\hline \multicolumn{6}{|l|}{$\begin{array}{l}\text { 7. ¿Organizás vos mismo cómo viajar para ir a las consultas } \\
\text { médicas? }\end{array}$} \\
\hline \multicolumn{6}{|l|}{$\begin{array}{l}\text { 8. ¿Te ocupás vos mismo de consultar al médico si observás } \\
\text { cambios inusuales en tu salud (por ejemplo: reacciones alérgicas, } \\
\text { etc.)? }\end{array}$} \\
\hline \multicolumn{6}{|l|}{$\begin{array}{l}\text { 9. ¿Sabés realizar vos mismo los trámites para obtener una } \\
\text { cobertura médica si perdés la actual? }\end{array}$} \\
\hline \multicolumn{6}{|l|}{ 10. ¿Sabés qué beneficios cubre tu obra social, prepaga, etc.? } \\
\hline \multicolumn{6}{|l|}{$\begin{array}{l}\text { 11. ¿Manejás tu propio dinero y te encargás de los gastos de la casa } \\
\text { (por ejemplo, utilizás tarjeta de débito, crédito, etc.)? }\end{array}$} \\
\hline \multicolumn{6}{|l|}{ Seguimiento de los problemas de salud } \\
\hline \multicolumn{6}{|l|}{$\begin{array}{l}\text { 12. ¿Podés completar vos mismo algún formulario relacionado con } \\
\text { tu historia clínica, incluida la lista de tus alergias? }\end{array}$} \\
\hline \multicolumn{6}{|l|}{$\begin{array}{l}\text { 13. ¿Tenés anotados tus turnos médicos y demás citas de alguna } \\
\text { manera (agenda, lista, celular, etc.)? }\end{array}$} \\
\hline \multicolumn{6}{|l|}{$\begin{array}{l}\text { 14. ¿Realizás vos mismo una lista de preguntas antes de ir a la } \\
\text { consulta médica? }\end{array}$} \\
\hline \multicolumn{6}{|l|}{$\begin{array}{l}\text { 15. ¿Recibis ayuda económica a través de alguna institución } \\
\text { (subsidio, pensión, apoyo, etc.)? }\end{array}$} \\
\hline \multicolumn{6}{|l|}{ Comunicación con los profesionales } \\
\hline \multicolumn{6}{|l|}{ 16. ¿Le contás al médico o a la enfermera lo que te pasa? } \\
\hline \multicolumn{6}{|l|}{$\begin{array}{l}\text { 17. ¿Respondés vos mismo las preguntas que te hacen el médico, la } \\
\text { enfermera u otra persona del equipo de salud? }\end{array}$} \\
\hline \multicolumn{6}{|l|}{ Manejo de las actividades cotidianas } \\
\hline \multicolumn{6}{|l|}{ 18. ¿Colaborás con la planificación o preparación de las comidas? } \\
\hline \multicolumn{6}{|l|}{$\begin{array}{l}\text { 19. ¿Mantenés ordenado tu cuarto ylo casa o levantás la mesa y/o } \\
\text { lavás los platos después de comer? }\end{array}$} \\
\hline $\begin{array}{l}\text { 20. ¿Vas a hacer compras a los negocios del barrio (por ejemplo: } \\
\text { almacén, farmacia, etc.)? }\end{array}$ & & & & & \\
\hline
\end{tabular}

(C) Wood, Sawicki, Reiss \& Livingood, 2012 